\title{
SPACES OF MEASURABLE TRANSFORMATIONS ${ }^{1}$
}

BY ROBERT J. AUMANN

Communicated by Paul R. Halmos, February 27, 1960

By a space we shall mean a measurable space, i.e. an abstract set together with a $\sigma$-ring of subsets, called measurable sets, whose union is the whole space. The structure of a space will be the $\sigma$-ring of its measurable subsets. A measurable transformation from one space to another is a mapping such that the inverse image of every measurable set is measurable.

Let $X$ and $Y$ be spaces, $F$ a set of measurable transformations from $X$ into $\cdot Y$, and $\phi_{F}: F \times X \rightarrow Y$ the natural mapping defined by $\phi_{F}(f, x)=f(x)$. A structure $R$ on $F$ will be called admissible if $\phi_{F}$, considered as a mapping from the product space $(F, R) \times X$ into $Y$, is a measurable transformation. ${ }^{2}$ It may not be possible to define an admissible structure on $F$; if it is, $F$ itself will also be called admissible. We are concerned with the problem of characterizing, for given $X$ and $Y$, the admissible sets $F$ and the admissible structures $R$ on the admissible sets.

The following three theorems may be established fairly easily:

TheOREM A. A set consisting of a single measurable transformation is admissible.

Theorem B. A subset of an admissible set is admissible. Indeed, if $G \subset F, R$ is an admissible structure on $F$, and $R_{G}$ is the subspace structure on $G$ induced by $R$, then $R_{G}$ is admissible on $G$.

THEOREM C. The union of denumerably many admissible sets is admissible. Indeed, if $F=\bigcup_{i=1}^{\infty} F_{i}$ and $R_{1}, R_{2}, \cdots$ are admissible structures on $F_{1}, F_{2}, \cdots$ respectively, then the structure $R$ on $F$ generated by the members of all the $R_{i}$ is admissible on $G$.

Much more can be said if $X$ and $Y$ are assumed to be separable, i.e. to have countably generated structures. ${ }^{4}$ To state our theorems in this case we first define the concept of Banach class, closely related to that of Baire class. Let $\mathfrak{A}$ be an arbitrary class of meas-

1 The author is much indebted to Professor P. R. Halmos, who suggested a number of significant improvements in the complete version of this note.

${ }^{2}(F, R)$ is the space whose underlying abstract set is $F$ and whose structure is $R$.

${ }^{3} R_{G}$ consists of all intersections of $G$ with members of $R$.

${ }^{4}$ The term is used by analogy with its topological use. We will also use the term "separable structure," meaning a countably generated structure. 
urable subsets of $X$. For each denumerable ordinal number $\alpha \geqq 1$, we define classes $P_{\alpha}(\mathfrak{H})$ and $Q_{\alpha}(\mathfrak{H})$ inductively as follows: $Q_{1}(\mathfrak{H})$ consists of all denumerable unions of members of $\mathfrak{A}$, and $P_{1}(\mathfrak{H})$ consists of all complements of members of $Q_{1}(\mathfrak{R})$; supposing $Q_{\beta}(\mathfrak{R})$ and $P_{\beta}(\mathfrak{H})$ to have been defined for all $\beta<\alpha$, we define $Q_{\alpha}(\mathfrak{H})$ $=Q_{1}\left(\bigcup_{\beta<\alpha} P_{\beta}(\mathfrak{H})\right)$ and $P_{\alpha}(\mathfrak{H})=P_{1}\left(\bigcup_{\beta<\alpha} P_{\beta}(\mathfrak{H})\right) . Q_{\alpha}(\mathfrak{H}) \cup P_{\alpha}(\mathfrak{H})$ is the set of all subsets of $X$ which can be "reached from $\mathfrak{A}$ " by performing at most $\alpha$ operations, where each operation consists of forming a denumerable union and a complement. If $\mathfrak{A}$ generates the structure of $X$, then the union (over $\alpha$ ) of all the $Q_{\alpha}(\mathfrak{H})$ (or of the $P_{\alpha}(\mathfrak{H})$ ) is the set of all measurable subsets of $X$. If $\mathfrak{B}$ is a class of measurable subsets of $Y$ and $\alpha \geqq 0$ is a denumerable ordinal number, then we define $L_{\alpha}(\mathfrak{A}, \mathfrak{B})$ to be the set of all functions $f: X \rightarrow Y$ such that for all $B \in Q_{1}(\mathfrak{B}), f^{-1}(B) \in Q_{\alpha+1}(\mathfrak{C})$. If $X$ and $Y$ are separable and $\mathfrak{A}$ and $\mathfrak{B}$ are denumerable generating sets for their respective structures, then the union (over $\alpha$ ) of all the $L_{\alpha}(\mathfrak{A}, \mathfrak{B})$ is the set of all measurable transformations from $X$ into $Y$. It will be denoted $Y^{X}$. In this case $L_{\alpha}(\mathfrak{A}, \mathfrak{B})$ is called the Banach class $^{5}$ of order $\alpha$ for ( $\left.\mathfrak{A}, \mathfrak{B}\right)$. A subset $F$ of $Y^{\boldsymbol{X}}$ is said to be of bounded Banach class if there is an $\alpha$ and denumerable generating sets $\mathfrak{A}, \mathfrak{B}$ such that $F \subset L_{\alpha}(\mathfrak{A}, \mathfrak{B})$. It is important to note that the definition of bounded Banach class is independent of the choice of $\mathfrak{A}$ and $\mathfrak{B}$, i.e. that if $F \subset L_{\alpha}(\mathfrak{A}, \mathfrak{B})$, then for any other generating pair $\mathfrak{A}^{\prime}, \mathfrak{B}^{\prime}$, there is an $\alpha^{\prime}$ such that $F \subset L_{\alpha^{\prime}}\left(\mathfrak{H}^{\prime}, \mathfrak{B}^{\prime}\right)$. If $X$ and $Y$ are separable metric spaces and $Y$ is pathwise connected, then the Banach classes coincide with the Baire classes (for appropriate choice of $\mathfrak{A}$ and $\mathfrak{B}$ ).

Theorem D. If $X$ and $Y$ are separable, then $F$ is admissible if and only if it is of bounded Banach class.

THEOREM E. If $X$ and $Y$ are separable, then every admissible subset of $Y^{X}$ has a separable admissible structure.

A space $Z$ and its structure are called regular if for all $x, y \in Z$, there is a measurable set in $Z$ containing $x$ but not $y$. It is known (cf. [2]) that a space is separable and regular if and only if it is isomorphic $^{6}$ to a subspace of $I$, where $I$ denotes the unit interval $[0,1]$ with the usual Borel structure.

TheOREM $F$. If $X$ and $Y$ are separable and regular, then every admissible subset of $Y^{X}$ has a separable and regular admissible structure.

${ }^{5}$ Because of the work that Banach [1] did in characterizing these classes.

${ }^{6}$ Two spaces are said to be isomorphic if there is a $1-1$ correspondence between them that preserves measurability (in both directions). 
The natural admissible structure on a given admissible set $F$ is defined to be the smallest admissible structure on $F$, if it exists. Alternatively, it may be defined to be the intersection of all the admissible structures on $F$, in case this is admissible. Not every admissible set need have a natural admissible structure; the counterexample is due to P. R. Halmos.

If $a \in X$ and $B \subset Y$, define $F(a, B)=\{f: f \in F, f(a) \in B\}$. It is not hard to prove that if $B$ is measurable and $a$ is arbitrary, then every admissible structure on $F$ must contain $F(a, B)$. A "converse" would be that the structure generated by the $F(a, B)$ is admissible, and it would follow that it is also natural.

THEOREM G. If $X$ and $Y$ are separable metric spaces and $F$ contains continuous functions only, then $F$ has a natural admissible structure, which is generated by the set of all $F(a, B)$, where $B$ is measurable and $a$ is arbitrary.

We now give some applications. A space is said to have the discrete structure if every subset is measurable. Let $J$ be the space consisting of 0 and 1 only, and $K$ the space of all positive integers, both with the discrete structure. If $X$ is an arbitrary space, then $X^{J}$ and $X^{K}$ are both admissible, and possess natural admissible structures which make them isomorphic to $X \times X$ and $X_{i=1}^{\infty} X_{i}$ respectively, where the $X_{i}$ are copies of $X$. In particular, $J^{K}$ is admissible and has a natural admissible structure which makes it isomorphic to $I$. These results are relatively trivial or at least easily derivable from known results.

The situation changes when we pass to exponent spaces with nondiscrete structures. For example, $J^{I}$ may be considered the set of all measurable subsets of $I$. It is not itself admissible. The set of all open subsets of $I$ is admissible, as is the set of all closed subsets, the set of all $G_{\delta}$, etc. In general, a subset $F$ of $J^{I}$ is admissible if and only if all members of $F$ can be constructed from the open subsets of $I$ by taking denumerable unions and intersections at most $\alpha$ times, where $\alpha$ is an arbitrary denumerable ordinal number (which is fixed for given $F$, but may differ for different $F$ ). I do not know whether or not every admissible subset of $J^{I}$ has a natural admissible structure, but if $F$ is admissible, then we may endow it with an admissible structure in such a way so that it will be isomorphic to a subset of $I$.

$I^{I}$ is not admissible. The set of all continuous functions from $I$ into $I$ is admissible; more generally, a necessary and sufficient condition that a subset $F$ of $I^{I}$ be admissible is that there exist a denumerable ordinal number $\alpha$ such that all members of $F$ are of Baire class $\alpha$ 
at most. The set $H$ of all continuous functions from $I$ into $I$ has a natural admissible structure; it is the Borel structure of $H$ when considered as a metric space (in the uniform convergence topology). Again, I do not know whether or not every admissible subset of $I^{I}$ has a natural admissible structure, but if $F$ is admissible, we may endow it with an admissible structure in such a way so that it will be isomorphic to a subset of $I$.

The above theory may be applied to give a generalization of Kuhn's theorem [3] about optimal behavior strategies in games of perfect recall, to games in which there may be a continuum of alternatives at some of the moves.

A fuller account of the theory outlined above, together with proofs, will be published elsewhere.

\section{REFERENCES}

1. S. Banach, Über analytisch darstellbare Operationen in abstrakten Räumen, Fund. Math. vol. 17 (1931) pp. 283-295.

2. G. W. Mackey, Borel structures in groups and their duals, Trans. Amer. Math. Soc. vol. 85 (1957) pp. 134-165.

3. H. W. Kuhn, Extensive games and the problem of information, Contributions to the Theory of Games II, Princeton University Press, 1953, pp. 245-266.

HEBREW UNIVERSITY, JERUSALEM, ISRAEL 